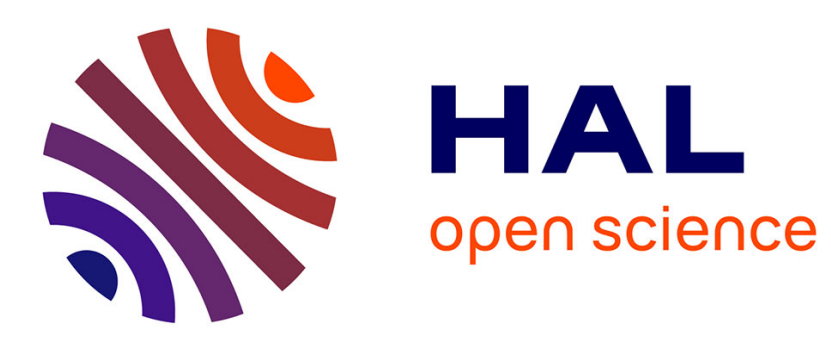

\title{
Relay Control Design using Attractive Ellipsoids Method
}

Andrey Polyakov, Laurentiu Hetel, Christophe Fiter

\section{To cite this version:}

Andrey Polyakov, Laurentiu Hetel, Christophe Fiter. Relay Control Design using Attractive Ellipsoids Method. 56th IEEE Conference on Decision and Control, Dec 2017, Melbourne, Australia. hal01589813

\section{HAL Id: hal-01589813 \\ https://hal.inria.fr/hal-01589813}

Submitted on 19 Sep 2017

HAL is a multi-disciplinary open access archive for the deposit and dissemination of scientific research documents, whether they are published or not. The documents may come from teaching and research institutions in France or abroad, or from public or private research centers.
L'archive ouverte pluridisciplinaire HAL, est destinée au dépôt et à la diffusion de documents scientifiques de niveau recherche, publiés ou non, émanant des établissements d'enseignement et de recherche français ou étrangers, des laboratoires publics ou privés. 


\title{
Relay Control Design using Attractive Ellipsoids Method
}

\author{
Andrey Polyakov, Laurentiu Hetel and Christophe Fiter
}

\begin{abstract}
Based on the attractive ellipsoids method the problem of rejection of both matched and mismatched exogenous disturbances is studied for a linear plants controlled by the generalized relays. Some existing results about local stabilizability as well as matching condition are refined for the system under consideration. The procedure of robust control design for optimal rejection of bounded exogenous disturbances is proposed. The theoretical results are supported with numerical simulations.
\end{abstract}

\section{INTRODUCTION}

Stabilization (or set-point tracking) is the classical control problem. Attractive Ellipsoids Method (AEM) has been developed for solving this problem for plants operating under uncertainty conditions (e.g. unknown but bounded exogenous disturbances) [1], [2], [3], [4], [5]. It is based on minimization of a simple set-theoretic criterion. Namely, it minimizes the attractive (and/or invariant) ellipsoid, which describes the stabilization error of the closed-loop system in the steady state. AEM is well developed for both linear [1], [2] and nonlinear control systems [3], [4], which allow the control input to vary continuously. However, to the best of our knowledge, attractive ellipsoids method has never been presented before for a class of the so-called (generalized) relay control systems [6], [7], [8], [9], [10], which allow the control input to take discrete values from a given admissible set. Such models appears in power electronics [11], in control of the separated airflow [12], in process control [13], etc.

Sliding mode is the main operation mode of variable structure (in particular, relay) control systems [8], [14]. The main advantage of the sliding mode control is its (at least theoretical) robustness with respect to the so-called matched disturbance. The matching (or invariance) condition [15], [8] specifies the case when the control may completely reject exogenous disturbances, so it guarantees robust asymptotic stability of the origin (set-point) of the closed-loop system. This condition is not well established for the relay control system. Indeed, in this paper we show that the conventional matching condition may be just necessary for existence of the relay control rejecting the disturbances, for example, if all admissible values of the relay control belongs to a certain subspace or a half space. So we need to refine slightly the matching condition in order to take into account the fact that the relay control may take only predefined set of values.

This research is partially supported by ANR ROCC-SYS (ANR-14-CE270008, "Robust Control of Cyber-Phisical Systems").

Andrey Polyakov is with Inria Lille-Nord Europe, France, (e-mail: andrey.polyakov@inria.fr).

Laurentiu Hetel is with CNRS CRIStAL, France, (e-mail: laurentiu.hetel@ec-lille.fr).

Christophe Fiter is with Université Lille 1, France, (e-mail: christophe.fiter@univ-lille1.fr)
In this paper we follow the convex embedding methodology proposed in [10]. It assumes the existence of a stabilizing controller, which locally (around the origin) can be embedded into a convex hull of all admissible values of the relay control. In [10], [16] to guarantee this property it was assumed that zero belongs to the interior of the mentioned convex hull. This paper is aimed at relaxation of this assumption, in particular zero may belong to a relative interior of the convex hull. The corresponding sufficient stabilizability condition is presented by Linear Matrix Inequalities (LMIs) using the fact that the convex hall of the finite set of vectors is, in fact, a convex polyhedron.

Finally, we develop Attractive Ellipsoids Method in order to propose a procedure of robust control design, which optimally rejects both matched and mismatched bounded exogenous disturbances. The corresponding design procedure is formalized as a parametrized Semi-Definite Programming (SDP) problem. It allows us both to reduce the disturbances effects and to maximize the domain of attraction. A possible way of solving this optimization problem is briefly discussed. It needs to combine an SDP solver with a gradient-free optimization procedure applied for two scalar parameters.

The paper is organized as follows: The next section presents problem statement and basic assumptions. Section III studies with the local stabilizability of linear plant by means of generalized relay control in the disturbance-free case. Section IV refines the matching condition and presents the control law that allows us to reject (completely) the matched exogenous disturbance. Section V develops AEM for generalized relay systems. Finally, the numerical examples and concluding remarks are given.

\section{Notation:}

- $\mathbb{R}$ is the field of real numbers, $\mathbb{R}_{+}=\{x \in \mathbb{R}: x \geq 0\}$; $\mathbb{N}$ is the set of natural numbers;

- $\mathcal{B}(\varepsilon)=\left\{x \in \mathbb{R}^{n}:\|x\|<\varepsilon\right\}$ is the ball of the radius $\varepsilon>0$;

- $\operatorname{int}(\Omega)$ denotes the interior of the set $\Omega \subset \mathbb{R}^{n}$;

- $\operatorname{span}\{\Omega\}$ denotes the linear hull of the set $\Omega \subset \mathbb{R}^{n}$, but the notation $c o\{\Omega\}$ is utilized for its convex hull;

- range $(M)$ denotes the column space of the matrix $M \in$ $\mathbb{R}^{n \times m}$ (i.e. the span of column vectors of $M$ );

- $\operatorname{rank}(M)$ denotes the rank of the matrix $M \in \mathbb{R}^{n \times m}$;

- $I_{n} \in \mathbb{R}^{n \times n}$ is the identity matrix;

- $B^{+} \in \mathbb{R}^{m \times n}$ denotes the left pseudo inverse matrix to $B \in \mathbb{R}^{n \times m}$ with $\operatorname{rank}(B)=m$, i.e. $B^{+}=\left(B^{T} B\right)^{-1} B^{T}$;

- the inequality $P>0$ for $P \in \mathbb{R}^{n \times n}$ means that $P$ is positive definite symmetric matrix. 


\section{Problem Statement}

Let us consider the model of a control system described by the ordinary differential equation (ODE):

$$
\dot{x}(t)=A x(t)+B u(t)+D d(t), \quad t \in \mathbb{R}_{+},
$$

where

- $x(t) \in \mathbb{R}^{n}$ is the state vector,

- $u(t) \in \mathbb{R}^{m}$ is the vector of control inputs,

- $A \in \mathbb{R}^{n \times n}$ is the system matrix,

- $B \in \mathbb{R}^{n \times m}$ is the matrix of control gains,

- the locally measurable function $d: \mathbb{R} \rightarrow \mathbb{R}^{r}$ describes the exogenous disturbances and $D \in \mathbb{R}^{n \times r}$.

We study the considered system under assumptions given below. First, we specify plant model.

Assumption 1:

- The matrices $A, B$ and $D$ are assumed to be known.

- The matrix $B$ is of full column $\operatorname{rank}$, i.e. $\operatorname{rank}(B)=$ $m \leq n$.

- The pair $(A, B)$ is controllable (or at least stabilizable).

- The whole state vector $x$ can be measured and utilized for control purposes.

Next, we describe the relay nature of the control.

Assumption 2:

- The control input u is assumed to be a generalized relay taking values from a given discrete set:

$$
u(t) \in \mathcal{U}:=\left\{v_{1}, v_{2}, \ldots, v_{N}\right\}, \quad v_{i} \in \mathbb{R}^{m}, \quad t \in \mathbb{R}_{+},
$$

where $N$ is a natural number.

- In addition, we assume that

$$
0 \in \operatorname{co}\{\mathcal{U}\} \subset \mathbb{R}^{m} .
$$

Finally, we restrict the class of the exogenous disturbances.

Assumption 3: The disturbance function $d$ is assumed to be locally measurable and uniformly bounded as follows

$$
d^{\top}(t) Q d(t) \leq 1, \quad t \in \mathbb{R}_{+},
$$

where $0<Q=Q^{\top} \in \mathbb{R}^{r \times r}$.

It is worth stressing that in Assumption 2 we relax the usual assumption $\mathbf{0} \in \operatorname{int}\{c o\{\mathcal{U}\}\}$ (see e.g. [10], [16] for the details). This relaxation is useful in many practical applications as well as for the case of switched systems, when the admissible control values are the vertexes of the unit simplex [17].

All claims given below assume by default that the presented assumptions hold.

The control aim is to stabilize (at least locally) the origin of the system (1) and to minimize the effect of disturbances.

Attractive Ellipsoids Method [1], [3], [2], [4] is supposed to be utilized for this purpose.

Being motivated by [10] in this paper we study the problem of robust stabilization via admissible relay switching law of the form

$$
u(t) \in u_{r}(x(t))=\underset{v \in \mathcal{U}}{\operatorname{argmin}} \Gamma^{\top}(x(t)) v,
$$

where $\Gamma: \mathbb{R}^{n} \rightarrow \mathbb{R}^{m}$ is a continuous vector-valued function.

The inclusion in (5) indicates that argmin is not unique in general case. In particular, if $m=1$ and $\mathcal{U}=\{-1,1\}$ then $u_{r}(x)=-\overline{\operatorname{sign}}[\Gamma(x)]$ similarly to the sliding mode control [18], where

$$
\overline{\operatorname{sign}}[\rho]=\left\{\begin{array}{ccc}
1 & \text { if } & \rho>0, \\
-1 & \text { if } & \rho<0, \\
\{-1,1\} & \text { if } & \rho=0 .
\end{array}\right.
$$

As we will see further, this configuration includes as a particular case the classical sliding control generated by sign functions. This control configuration may also be related to the simplex method in [19], [20] and to the stabilization of switched affine systems [21], [11].

Filippov theory of differential equations with discontinuous right-hand sides [22] is utilized in order to take into account the discontinuity of the control law, namely, for stability analysis of the closed-loop system we consider the differential inclusion

$$
\dot{x}(t) \in A x(t)+B c o\left\{\underset{v \in \mathcal{U}}{\operatorname{argmin}} \Gamma^{\top}(x(t)) v\right\}+D d(t) .
$$

Since the function $\Gamma$ is continuous on $\mathbb{R}^{n}$ then $c o\left\{\operatorname{argmin}_{v \in \mathcal{U}} \Gamma(\cdot) v\right\}$ is upper semicontinuous. The setvalued function in the right-hand side of the latter system satisfies the sufficient conditions of existence of Filippov solutions [22], namely, it is nonempty, compact, convexvalued and upper semicontinuous.

Remark 1: Note that in order to define the control input according to the formula (5) we just need to find a minimum of $\Gamma^{\top}(x(t)) v$ over finite set values $v \in \mathcal{U}$. This operation does not need applying any complicated optimization procedure. Just $N$ scalar products $\Gamma^{\top}(x(t)) v_{i}, i=1, \ldots, N$ must be calculated and compared at each instant of time.

\section{LocAl StabilizABILITy OF GENERALIZEd RELAY CONTROL SYSTEM}

In [10], [16] the inclusion $\mathbf{0} \in \operatorname{int}\{c o(\mathcal{U})\}$ was necessary for the proof of existence of some locally stabilizing feedback. The next theorem slightly relaxes this condition showing (implicitly) that $\mathbf{0}$ may belong just to the relative interior of $\operatorname{co}(\mathcal{U})$.

Theorem 1: Let $\mathbf{0} \in \operatorname{co}\{U\}$ and the LMI

$$
\begin{gathered}
A X+X A^{\top}+B Y+Y^{\top} B^{\top}<0, \quad X>0, \\
X=X^{\top} \in \mathbb{R}^{n \times n}, \quad Y \in \mathbb{R}^{m \times n}
\end{gathered}
$$

has a solution with respect to the variable $X, Y$ such that

$$
\operatorname{range}(Y) \subset \Pi \text {, }
$$

where $\Pi$ is the positive convex cone defined as follows

$$
\Pi:=\{\rho z: \rho>0, z \in \operatorname{co}(\mathcal{U})\} .
$$

Then the disturbance-free $(d=0)$ system (1) is locally asymptotically stabilizable by means of the feedback (5) with

Proof.

$$
\Gamma(x)=B^{\top} X^{-1} x .
$$


On the one hand, since $\mathbf{0} \in c o(U)$ then due to convexity we have

$$
\mathbf{0} \in \rho_{1} \operatorname{co}(U) \subset \rho_{2} \operatorname{co}(U)
$$

provided that $0<\rho_{1} \leq \rho_{2}$. Hence, $\rho \operatorname{co}(U)$ becomes $\Pi$ as $\rho \rightarrow+\infty$.

On the other hand, for any $\varepsilon>0$ we have

$$
Y \mathcal{B}(\varepsilon) \subset \operatorname{range}(Y),
$$

where $\mathcal{B}(\varepsilon)=\left\{x \in \mathbb{R}^{n}:\|x\|<\varepsilon\right\}$ is the ball of the radius $\varepsilon>0$. Since the set $Y \mathcal{B}(\varepsilon)$ is bounded then (8), (9) imply that there exists $\rho>0$ such that $Y \mathcal{B}(\varepsilon) \subset \rho \operatorname{co}(\mathcal{U})$ or equivalently

$$
Y \mathcal{B}(\varepsilon / \rho) \subset \operatorname{co}(\mathcal{U}) .
$$

The feasibility of LMI (7) implies existence of the continuous linear feedback $u_{c}(x)=Y X^{-1} x$, which stabilizes globally the system (1) (see, e.g. [23] for the details) and $2 x^{\top} X^{-1}\left(A x+B u_{c}(x)\right)<0$.

The inclusion

$$
u_{c}(x)=Y X^{-1} x \in \operatorname{co}(\mathcal{U}) \quad \text { if } \quad X^{-1} x \in \mathcal{B}(\varepsilon / \rho) .
$$

implies local stabilizability. Indeed, let the relay control law be defined by (5), (10). Applying the convex representation

$$
\begin{gathered}
u_{c}(x)=\sum_{i=1}^{N} \alpha_{i}(x) v_{i} \in \operatorname{co}\{\mathcal{U}\} \quad \text { if } \quad X^{-1} x \in \mathcal{B}(\varepsilon / \rho), \\
\alpha_{i}(x) \geq 0: \sum_{i=1}^{N} \alpha_{i}(x)=1,
\end{gathered}
$$

we derive (using (6))

$$
\begin{gathered}
\frac{d}{d t}\left(x^{\top} X^{-1} x\right) \leq 2 x^{\top} X^{-1}\left(A x+B u_{c}(x)\right)+ \\
\sup _{z \in c o\left\{\operatorname{argmin}_{v \in \mathcal{U}} \Gamma^{T}(x(t)) v\right\}} 2 x^{\top} X^{-1} B\left(z-u_{c}(x)\right)
\end{gathered}
$$

along the trajectories of the closed-loop system (1), (5), (10) (with $d=0$ ). Since for any $z_{0} \in \operatorname{argmin}_{v \in \mathcal{U}} \Gamma^{\top}(x(t)) v$ one has $x^{\top} X^{-1} B z_{0} \leq x^{\top} X^{-1} B v_{i}$ for all $i \in\{1, \ldots N\}$ then

$$
x^{\top} X^{-1} B z \leq x^{\top} X^{-1} B v_{i}
$$

for all $z \in c o\left\{\operatorname{argmin}_{v \in \mathcal{U}} \Gamma^{\top}(x(t)) v\right\}$. Taking into account $\sum_{i=1}^{N} \alpha_{i}(x)=1$ we obtain

$x^{\top} X^{-1}\left(B z-B u_{c}(x)\right)=\sum_{i=1}^{N} x^{\top} X^{-1} B \alpha_{i}(x)\left(z-v_{i}\right) \leq 0$

if $z \quad \in \quad c o\left\{\operatorname{argmin}_{v \in \mathcal{U}} \Gamma^{\top}(x) v\right\} . \quad$ Therefore, $\frac{d}{d t}\left(x^{\top}(t) X^{-1} x(t)\right)<0$ along the trajectories of the closed-loop system (1), (5), (10) (with $d=0$ ) and $V: \mathbb{R}^{n} \rightarrow \mathbb{R}_{+}$defined as $V(x)=x^{\top} X^{-1} x$ is the Lyapunov function.

Remark 2: It is worth stressing that the sufficient condition (7), (8) may become necessary in some particular cases, for example, if $m=1$ and the matrix $A$ has only one eigenvalue in the right complex half plane, but all other eigenvalues are placed in the left one. Indeed, this claim becomes obvious if we transform the original system to the real Jordan form.
The next corollary uses the fact that the convex hull of $\mathcal{U}$ is, in fact, a convex polyhedron in $\mathbb{R}^{m}$, which can be degenerated (i.e. $\operatorname{dim}(\operatorname{span}\{\mathcal{U}\}) \leq m$ ) in the general case.

Corollary 1: Let $X \in \mathbb{R}^{n \times n}, Y \in \mathbb{R}^{n \times m}$ satisfy (7) and

$$
\operatorname{co}(\mathcal{U})=\left\{\xi \in \mathbb{R}^{m}: h_{i}^{\top} \xi \leq q_{i}, i=1,2, \ldots, \tilde{N}\right\},
$$

where the hyperplanes $h_{i}^{\top} \xi=q_{i}, h_{i} \in \mathbb{R}^{m}, q_{i} \in \mathbb{R}_{+}, i=$ $1,2, \ldots, \tilde{N}$ define the faces of the polyhedron co(U). If LMIs

$$
\left(\begin{array}{cc}
X & Y^{\top} h_{i} \\
h_{i}^{\top} Y & \alpha q_{i}^{2}
\end{array}\right) \geq 0, \quad \alpha \in \mathbb{R}_{+}, \quad i=1,2, \ldots, \tilde{N},
$$

are feasible then the origin of the closed-loop system (1), (5), (10) (with $d=0$ ) is locally asymptotically stable and the ellipsoid

$$
\mathcal{E}\left(X, \alpha^{-1}\right)=\left\{x \in \mathbb{R}^{n}: x^{\top} X^{-1} x \leq \alpha^{-1}\right\}
$$

belongs to its domain of attraction.

Proof. If $q_{i}=0$ then the LMI (12) hold only if $h_{i}^{T} Y=0$ this immediately means $h_{i}^{T} Y X^{-1} x \leq q_{i}=0$.

For $q_{i}>0$ one has The LMI (12) implies (due to the Schur complement)

$$
\frac{x^{T} X^{-1} Y^{T} h_{i} h_{i}^{T} Y X^{-1} x}{q_{i}^{2}} \leq \alpha x^{T} X^{-1} x \leq 1 \text { if } x \in \mathcal{E}\left(X, \alpha^{-1}\right) .
$$

Hence, $h_{i}^{T} Y X^{-1} x \leq q_{i}$ and $Y X^{-1} x \in c o(\mathcal{U})$ if $x \in$ $\mathcal{E}\left(X, \alpha^{-1}\right)$. The latter obviously implies (8). Finally, since $x^{T} X^{-1} x$ defines the Lyapunov function $\left(x^{T} X^{-1} x>0\right.$ and $\frac{d}{d t}\left(x^{T} X^{-1} x\right)<0$ for $x \neq 0$ ) to the closed-loop system (see the proof of Theorem 1) then its level set $\mathcal{E}\left(X, \alpha^{-1}\right)$ belongs to the domain of attraction.

\section{Rejection of Matched Disturbances}

This section refines the invariance condition [15] (matching condition [8]) known for sliding mode control systems.

Theorem 2: If the conditions (7), (8) hold and

$$
\text { range }(D) \subset \operatorname{range}(B Y) \text {, }
$$

then the origin of the closed-loop system (1), (4), (5), (10) is locally asymptotically stable provided that the disturbance function $d$ is sufficiently small (or, equivalently, $\lambda_{\min }(Q)$ is sufficiently large).

Proof. The condition (14) implies that

$$
\operatorname{Dd}(t)=B Y \gamma(t),
$$

for some measurable bounded function $\gamma: \mathbb{R}_{+} \rightarrow \mathbb{R}$ with $\sup \|\gamma\|$ proportional to sup $\|d\|$. In this case the feedback control

$$
\tilde{u}_{c}(t, x)=Y\left(X^{-1} x-\gamma(t)\right)
$$

globally stabilizes the system (1). Repeating the consideration of Theorem 1 for sufficiently small $\gamma$ we can show that $\tilde{u}_{c}(t, x) \in c o\{\mathcal{U}\}$ if $X^{-1} x \in \mathcal{B}(\varepsilon / \rho-\delta)$, where $\delta \in(0, \varepsilon / \rho)$ is proportional to sup $\|\gamma\|$, so the closed-loop system (1), (4), (5), (10) is locally asymptotically stable.

The conventional matching condition range $(D) \subset$ range $(B)$ obviously necessary but not sufficient for (14). 
Let $B^{+} \in \mathbb{R}^{m \times n}$ be pseudo inverse matrix to $B \in \mathbb{R}^{n \times m}$ such that $B^{+} B=I_{m}$. Under Assumption 1 such a matrix always exists and it can be defines as $B^{+}=\left(B^{T} B\right)^{-1} B^{T}$.

Corollary 2: Let

- $c o\{\mathcal{U}\}$ be defined by (11),

- $0 \in \operatorname{co}\{\mathcal{U}\}$,

- the LMI (7) is fulfilled,

- range $(D) \subset$ range $(B)$.

If there exist $\tau_{i} \in(0,1), i=1,2, \ldots, \tilde{N}$ such that the $L M I$

$$
\left(\begin{array}{cc}
\tau_{i} X & Y^{\top} h_{i} \\
h_{i}^{\top} Y & \alpha c_{i}
\end{array}\right) \geq 0, \quad \alpha>0,
$$

is feasible with

$$
c_{i}=q_{i}^{2}-\left(1-\tau_{i}\right)^{-1} h_{i}^{\top} B^{+} D Q^{-1} D^{\top}\left(B^{+}\right)^{\top} h_{i}
$$

then the origin of the closed-loop system (1), (4), (5), (10) is locally asymptotically stable and the ellipsoid (13) belongs to its domain of attraction.

Proof. The conventional matching condition range $(D) \subset$ range $(B)$ implies

$$
B u(t)+D d(t)=B\left(u(t)+B^{+} D d(t)\right)
$$

and $\tilde{u}_{c}(t, x)=Y X^{-1}-B^{+} D d(t)$. Since

$$
\begin{gathered}
0 \leq\left(\begin{array}{cc}
\tau_{i} X & Y^{\top} h_{i} \\
h_{i}^{\top} Y & \alpha c_{i}
\end{array}\right)=\left(\begin{array}{cc}
\tau_{i} X & Y^{\top} h_{i} \\
h_{i}^{\top} Y & \alpha q_{i}^{2}
\end{array}\right)- \\
\left(\begin{array}{c}
\mathbf{0} \\
D^{T}\left(B^{+}\right)^{\top} h_{i}
\end{array}\right)^{T} \frac{\alpha}{1-\tau_{i}} Q^{-1}\left(\begin{array}{c}
\mathbf{0} \\
D^{T}\left(B^{+}\right)^{\top} h_{i}
\end{array}\right)
\end{gathered}
$$

then applying Schur complement we derive

$$
\left(\begin{array}{ccc}
\tau_{i} X & \mathbf{0} & Y^{\top} h_{i} \\
\mathbf{0} & \frac{1-\tau_{i}}{\top^{\alpha}} Q & D^{\top}\left(B^{+}\right)^{\top} h_{i} \\
h_{i}^{\top} Y & h_{i}^{\top} B^{+} D & \alpha q_{i}^{2}
\end{array}\right) \geq 0 .
$$

Applying once more the Schur complement to the latter inequality (15) we conclude

$$
\frac{\left(\begin{array}{c}
X^{-1} x \\
-d(t)
\end{array}\right)^{\top}\left(\begin{array}{ll}
Y^{\top} & B^{+} D
\end{array}\right)^{\top} h_{i} h_{i}^{\top}\left(\begin{array}{ll}
Y & B^{+} D
\end{array}\right)\left(\begin{array}{c}
X^{-1} x \\
-d(t)
\end{array}\right)}{q_{i}^{2}} \leq
$$

Hence, Assumption 3 implies $\left(h_{i}^{\top}\left(Y X^{-1} x-B^{+} D d(t)\right)\right)^{2} \leq$ 1 if $x \in \mathcal{E}\left(X, \alpha^{-1}\right)$ and $\tilde{u}_{c}(t, x) \in \operatorname{co}\{\mathcal{U}\}$.

It is worth stressing that for $q_{i} \neq 0$ the LMI (15) is always feasible if the matrix $Q$ is sufficiently large (i.e. the disturbance function $d$ is sufficiently small). For $q_{i}=0$ it is feasible only if $h_{i}^{T} Y=0$ and $h_{i}^{T} B^{+} D=0$.

\section{Attractive Ellipsoids Method}

Optimization of an attractive ellipsoids is useful if range $(D) \notin \operatorname{range}(B)$, i.e. disturbances can be partly rejected. The next theorem adapts the attractive ellipsoids technique [1], [3] to the case of generalized relay system.

Theorem 3: Let the system of LMIs

$$
\begin{gathered}
A X+X A^{\top}+B Y+Y^{\top} B^{\top}+\beta X+\frac{D Q^{-1} D^{\top}}{\beta} \leq 0, \\
0<X=X^{\top} \in \mathbb{R}^{n \times n}, \quad Y \in \mathbb{R}^{m \times n}, \quad \beta \in \mathbb{R}_{+},
\end{gathered}
$$

and (15) be feasible for some $\beta \in \mathbb{R}_{+}$and $\alpha, \tau_{i} \in(0,1)$.

Then the origin of the closed-loop system (1), (5), (10) is locally practically stable with the domain of attraction $\mathcal{E}\left(X, \alpha^{-1}\right)$ and the attractive set $\mathcal{E}(X, 1)$, i.e.

$$
x(0) \in \mathcal{E}\left(X, \alpha^{-1}\right) \quad \Rightarrow \quad x(t) \rightarrow \mathcal{E}(X, 1) \text { as } t \rightarrow \infty .
$$

Proof. Let us consider the Lyapunov function defined as $V(x)=x^{T} X^{-1} x$. Its derivative along the trajectories of the closed-loop system admits the representation

$$
\begin{aligned}
\dot{V}(x)= & x^{\top} X^{-1}\left(\left(A+B Y X^{-1}\right) x+D d(t)\right) x \\
& +x^{\top} X^{-1} B\left(u(t)-Y X^{-1} x\right) .
\end{aligned}
$$

Since $Y X^{-1} x \in \operatorname{co}\{\mathcal{U}\}$ for $x \in \mathcal{E}(X, \alpha)$ due to (12) then repeating the last part of the proof of Theorem 1 we show

$$
x^{T} X^{-1} B\left(u(t)-Y X^{-1} x\right) \leq 0 .
$$

Therefore, we have

$$
\begin{gathered}
\dot{V}(x) \leq \\
\left(\begin{array}{c}
x \\
d(t)
\end{array}\right)^{\top}\left(\begin{array}{cc}
A X+X A^{\top}+B Y+Y^{T} B^{T} & D \\
D^{T} & 0
\end{array}\right)\left(\begin{array}{c}
x \\
d(t)
\end{array}\right)^{\top} \\
+\beta\left(x^{\top} X^{-1} x-x^{\top} X^{-1} x+d^{\top}(t) Q d(t)-d^{\top}(t) Q d(t)\right) .
\end{gathered}
$$

The LMI (16) and Assumption 3 imply

$$
\dot{V}(x) \leq-\beta V(x)+\beta
$$

if $x \in \mathcal{E}\left(X, \alpha^{-1}\right)$. Hence, if $x(0) \in \mathcal{E}\left(X, \alpha^{-1}\right)$ then

$$
V(x(t))=x^{\top}(t) X^{-1} x(t) \leq e^{-\beta t} V(x(0))+1-e^{-\beta t} \rightarrow 1
$$

as $t \rightarrow+\infty$.

To improve the control precision the attractive ellipsoid $\mathcal{E}(X, 1)$ must be minimized, but to increase the guaranteed attraction domain $\mathcal{E}\left(X, \alpha^{-1}\right)$ we need to minimize the parameter $\alpha$. These goals can be achieved by means of solving the optimization problem like

$$
J(X, Y, \alpha, \beta, \tau):=\alpha+\operatorname{trace}(X) \rightarrow \min _{\alpha, \beta, \tau, X, Y}
$$

subject to (16), (15) and $\alpha \in(0,1), \tau_{i}=\tau \in(0,1)$.

For any fixed $\beta$ and $\tau$ the latter problem becomes SDP (Semidefinite Programming Problem) [23], which can be solved using one of many existing toolboxes.

Let us introduce a functional

$$
\tilde{J}(\beta, \tau)=\min _{X, Y, \alpha} J(X, Y, \alpha, \beta, \tau) .
$$

To optimize the latter functional with respect to parameters $\alpha$ and $\tau$ we need to use some derivative free optimization algorithm (e.g. fminsearch command of MATLAB).

It is worth stressing that the functional $\tilde{J}$ is convex with respect to parameter $\beta \in \mathbb{R}_{+}$(see, [1]), so optimum with respected to $\beta$ can always be guaranteed. Result about convexity or non-convexity of $\tilde{J}$ with respect to the parameter $\tau$ is not established yet. So, it may have local minimum with respect to $\tau \in(0,1)$ and the search from different initial values of $\tau$ may need to be done in order to find a solution of the considered optimization problem. 


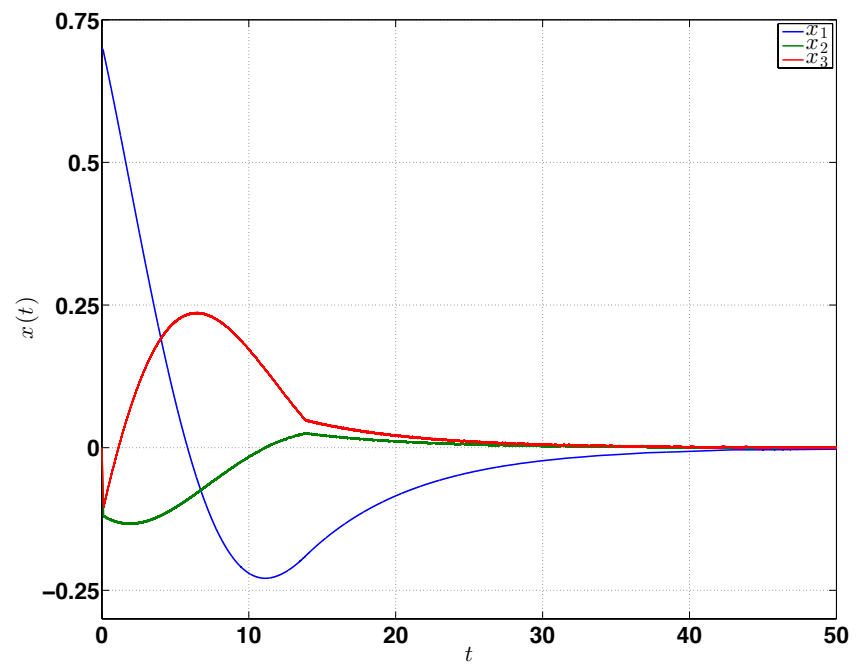

Fig. 1. Evolution of the system state of the closed-loop system (1), (5), (10) in the disturbance-free case

\section{NuMERICAL EXAMPLE}

Let us consider the system (1), (2) with

$$
\begin{gathered}
A=\left(\begin{array}{ccc}
0 & 1 & 0 \\
0 & -0.1 & 0.1 \\
0.2 & 0 & 0.1
\end{array}\right), B=\left(\begin{array}{ll}
0 & 0 \\
1 & 0 \\
0 & 1
\end{array}\right), \\
\mathcal{U}=\left\{\left(\begin{array}{l}
1 \\
1
\end{array}\right),\left(\begin{array}{l}
-1 \\
-1
\end{array}\right),\left(\begin{array}{l}
0 \\
1
\end{array}\right)\right\} .
\end{gathered}
$$

Obviously, $0 \notin \operatorname{int}\{c o\{U\}\}$, but $0 \in \operatorname{co}\{U\}$. The set $\operatorname{co}\{U\}$ admits the representation (11) with

$$
\begin{gathered}
h_{1}=\left(\begin{array}{c}
-1 \\
1
\end{array}\right), \quad q_{1}=0, \\
h_{2}=\left(\begin{array}{c}
1 \\
0
\end{array}\right), \quad q_{2}=1, \\
h_{3}=\left(\begin{array}{c}
1 \\
-2
\end{array}\right), \quad q_{3}=1 .
\end{gathered}
$$

\section{A. Disturbance-free Case}

Applying the LMI solver to the system of matrix inequalities (7), (12) we derive

$$
X=\left(\begin{array}{ccc}
93.8211 & -12.4188 & -23.8972 \\
-12.4188 & 36.2683 & 14.6596 \\
-23.8972 & 14.6596 & 76.9367
\end{array}\right),
$$

and

$$
\alpha=88.9532 .
$$

Due to Corollary 1 the closed-loop generalized relay system (1), (5), (10) is locally asymptotically stable and the ellipsoid (13) belongs to the domain of attraction of the origin.

Fig. 1 depicts the results of simulation of the closedloop relay system in the disturbance-free case with $x(0)=$ $(0.7,0,0)^{T}$. The simulation have been done using the explicit Euler method with step size 0.001 .

The numerical simulations also confirms the well-known fact that the sliding mode is the main operation regime of the

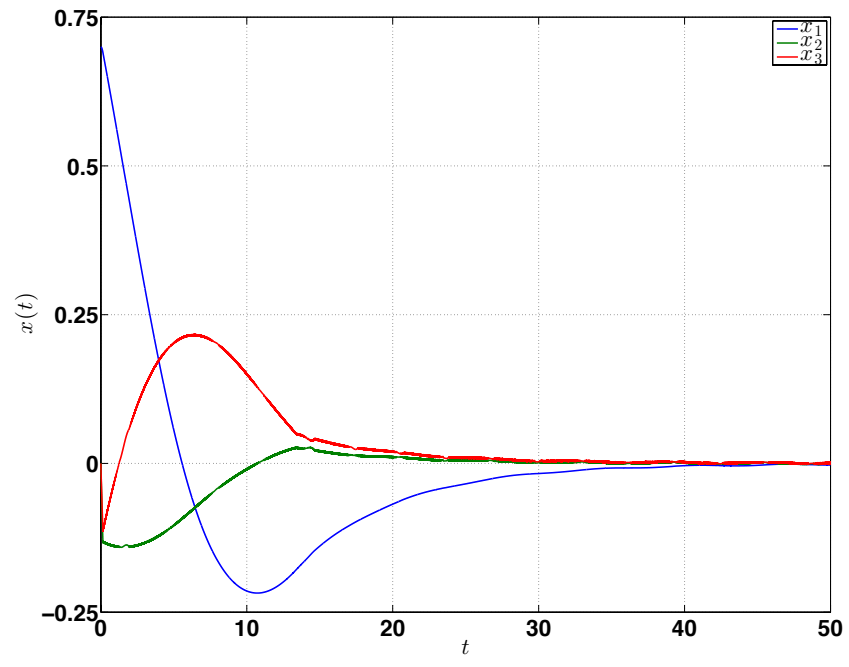

Fig. 2. Evolution of the system state of the closed-loop system (1), (5), (10) in the case of the matched disturbance $(d(t)=0.5 \sin (t))$

relay control system. The sliding (high frequency switching) mode in this system starts from the initial instant of time despite of the fact we do not define explicitly the sliding surface.

\section{B. Rejection of the matched disturbance}

Let us assume that

$$
D=\left(\begin{array}{l}
0 \\
1 \\
1
\end{array}\right)
$$

and the disturbance function $d$ is bounded as

$$
\left.d^{2}(t) \leq 0.25 \quad \text { (i.e. } Q=4\right) .
$$

The system of LMIs (7), (15) is feasible with $\tau=\tau_{i}=0.7$,

$$
X=\left(\begin{array}{ccc}
113.0549 & -17.1670 & -31.7816 \\
-17.1670 & 38.1731 & 8.9829 \\
-31.7816 & 8.9829 & 88.8233
\end{array}\right)
$$

and

$$
\alpha=236.5611
$$

Therefore, from Corollary 2 we deduce that the matrix $D$ satisfies the matching condition to the generalized relay system (1), (2), the closed-loop system (1), (5), (10) is locally asymptotically stable for all disturbances satisfying the condition (4) and the ellipsoid (13) belong to the domain of attraction of the origin.

Fig. 2 shows the simulation results for the case of the matched disturbances with $d(t)=0.5 \sin (t)$. They confirm the theoretically proven claim that the matched disturbances can be rejected completely. Similarly to the classical sliding mode control system, our closed-loop generalized relay control system is almost nonsensitive with respect to the matched perturbations. 


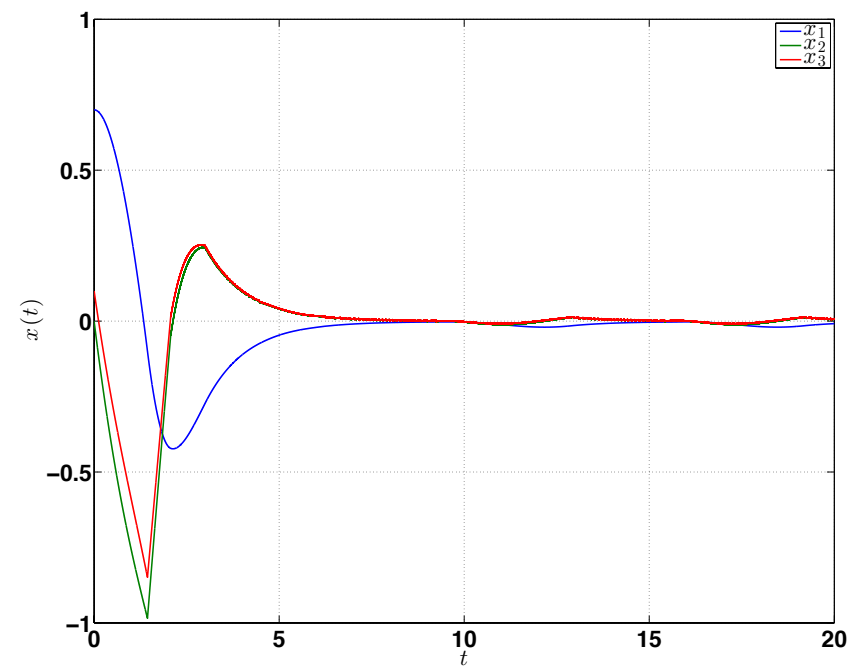

Fig. 3. Evolution of the system state of the closed-loop system (1), (5), (10) in the case of mismatched disturbances

\section{Reduction of mismatched disturbances}

The small variation of the matrix $D$ like

$$
D=\left(\begin{array}{c}
0 \\
1 \\
0.95
\end{array}\right)
$$

makes infeasible the system of LMIs (7), (15), despise of the fact that the classical matching(invariance) condition range $(D) \subset \operatorname{range}(B)$ remains true. Therefore, specific restrictions to the admissible values of the control inputs do not allow such disturbances to be rejected completely. We should use the method of attractive ellipsoid in order to minimize the effects of disturbance $d$ to the closed-loop system.

Solving the optimization problem (17) we derive (for $\tau=$ $\left.\tau_{i}=0.7\right)$

$$
X=\left(\begin{array}{ccc}
0.0094 & -0.0084 & -0.0086 \\
-0.0084 & 0.0322 & 0.0315 \\
-0.0086 & 0.0315 & 0.0310
\end{array}\right)
$$

and

$$
\alpha=0.9872, \quad \beta=1.81 .
$$

The simulation results for the mismatched case are given at Fig. 3. They confirm practical stability of the closed-loop system.

\section{CONCLUSIONS}

In this paper the three new results are presented:

- the sufficient condition of local stabilizability of relay control systems is revised;

- the matching (invariance) condition for relay control systems is revised;

- the attractive ellipsoids method is extended to the class of generalized relay control systems.

\section{REFERENCES}

[1] B. Polyak, P. Shcherbakov, and M. Topunov, "Invariant ellipsoids approach to robust rejection of persistent disturbances," in Proceedings of the IFAC World Congress, 2008, pp. 3976-3981.

[2] B. Polyak, M. Khlebnikov, and P. Shcherbakov, Control of Linear System under Exogeneous Disturbances (in Russian). Ledland, Moscow, 2013.

[3] S. Gonzalez-Garcia, A. Polyakov, and A. Poznyak, "Output linear controller for a class of nonlinear systems using the invariant ellipsoid technique, , 1160-1165," in American Control Conference, 2009.

[4] A. Poznyak, A. Polyakov, and V. Azhmyakov, Attractive Ellipsoids in Robust Control. Birkhauser, 2014.

[5] M. Mera, I. Salgado, and I. Chairez, "Robust control for state constrained uncertain systems: Attractive ellipsoid method approach," in 10th IFAC Symposium on Nonlinear Control Systems NOLCOS, 2016.

[6] I. Flugge-Lotz, Discontinuous automatic control. Princeton University Press, 1953.

[7] Y. Z. Tsypkin, Relay Control Systems. Cambridge University Press, 1984.

[8] V. I. Utkin, Sliding Modes in Control Optimization. Berlin: SpringerVerlag, 1992.

[9] L. Fridman, V. Strygin, and A. Polyakov, "Nonlocal stabilization via delayed relay control rejecting uncertainty in a time delay," International Journal of Robust and Nonlinear Control, 2004.

[10] L. Hetel, E. Fridman, and T. Floquet, "Variable structure control with generalized relays: A simple convex optimization approach," IEEE Transactions on Automatic Control, vol. 60(2), pp. 497-502, 2015.

[11] G. S. Deaecto, J. C. Geromel, F. S. Garcia, and J. A. Pomilio, "Switched affine systems control design with application to dc-dc converters," IET Control Theory \& Applications, vol. 4, no. 4, pp. 1201-1210, 2010.

[12] M. Feingesicht, C. Raibaudo, A. Polyakov, F. Kerhervé, and J.-P. Richard, "A bilinear input-output model with state-dependent delay for separated flow control," in European Control Conference 2016, Aalborg, 2016

[13] I. Boiko, Discontinuous control systems : frequency-domain analysis and design. Boston : Birkhauser, 2009.

[14] C. Edwards and S. Spurgeon, Sliding Mode Control: Theory and Applications. Taylor and Francis, 1998.

[15] B. Drazenovic, "The invariance conditions in variable structure systems," Automatica, vol. 5, no. 3, pp. 287-295, 1969.

[16] A. Polyakov and L. Hetel, "Relay control design for robust stabilization in a finite-time," IEEE Transactions on Automatic Control, 2017 (10.1109/TAC.2016.2591725),

[17] L. Hetel and E. Bernuau, "Local stabilization of switched affine systems," IEEE Transactions on Automatic Control, vol. 60, no. 4, pp. 1158-1163, 2015.

[18] V. Utkin, J. Guldner, and J. Shi, Sliding Mode Control in ElectroMechanical Systems. CRC Press, 2009.

[19] G. Bartolini, E. Punta, and T. Zolezzi, "Simplex sliding mode control of multi-input systems with chattering reduction and mono-directional actuators," Automatica, vol. 47, pp. 2433-2437, 2011.

[20] S. V. Bajda and D. B. Izosimov, "Vector method of design of sliding motion and simplex algorithms," Automation and Remote Control, vol. 46, pp. 830-837, 1985.

[21] P. Bolzern and W. Spinelli, "Quadratic stabilization of a switched affine system about a nonequilibrium point," in Proceeding of the 2004 American Control Conference, Boston, Massachusetts, USA, 2004.

[22] A. F. Filippov, Differential Equations with Discontinuous Righthand Sides. Kluwer Academic Publishers, 1988.

[23] S. Boyd, E. Ghaoui, E. Feron, and V. Balakrishnan, Linear Matrix Inequalities in System and Control Theory. Philadelphia: SIAM, 1994. 